\title{
Comparative epidemiology of influenza $A$ and $B$ viral infection in a subtropical region: a 7-year surveillance in Okinawa, Japan
}

\author{
Yoshikazu Iha ${ }^{1,2}$, Takeshi Kinjo ${ }^{*}$, Gretchen Parrott ${ }^{1}$, Futoshi Higa $^{3}$, Hideaki Mori ${ }^{4}$ and Jiro Fujita ${ }^{1}$
}

\begin{abstract}
Background: The epidemic patterns of influenza B infection and their association with climate conditions are not well understood. Influenza surveillance in Okinawa is important for clarifying transmission patterns in both temperate and tropical regions. Using surveillance data, collected over 7 years in the subtropical region of Japan, this study aims to characterize the epidemic patterns of influenza B infection and its association with ambient temperature and relative humidity, in a parallel comparison with influenza A.
\end{abstract}

Methods: From January 2007 until March 2014, two individual influenza surveillance datasets were collected from external sources. The first dataset, included weekly rapid antigen test (RAT) results from four representative general hospitals, located in the capital city of Okinawa. A nation-wide surveillance of influenza, diagnosed by RAT results and/or influenza-like illness symptoms, included the age distribution of affected patients and was used as the second dataset. To analyze the association between infection and local climate conditions, ambient temperature and relative humidity during the study period were retrieved from the Japanese Meteorological Agency website.

Results: Although influenza A maintained high number of infections from December through March, epidemics of influenza B infection were observed annually from March through July. The only observed exception was 2010, when the pandemic strain of 2009 dominated. During influenza B outbreaks, influenza patients aged 5 to 9 years old and 10 to 14 years old more frequently visited sentinel sites. Although both ambient temperature and relative humidity are inversely associated with influenza A infection, influenza B infection was found to be directly associated with high relative humidity.

Conclusion: Further studies are needed to elucidate the complex epidemiology of influenza B and its relationship with influenza A. In the subtropical setting of Okinawa, epidemics of influenza B infection occur from March to July following the influenza A epidemic, and primarily affect school-age children. These findings help to define unknown aspects of influenza B and can inform healthcare decisions for patients located outside temperate regions.

Keywords: Influenza B, Epidemics, Seasonality, School-age, Climate condition, Temperature, Humidity

\section{Background}

Influenza A virus mutates frequently to escape the host immune system and often causes epidemics. Historically, this had led to pandemics. Thus, influenza A infection is regarded as important to both medical and social concerns $[1,2]$. Alternatively, influenza B virus mutates less frequently than influenza A virus [3, 4]. Although some regional outbreaks are due to influenza $B$ virus [5-8], the

\footnotetext{
* Correspondence: t_kinjo@med.u-ryukyu.ac.jp

'Department of Infectious Diseases, Respiratory, and Digestive Medicine, Graduate School of Medicine, University of the Ryukyus, 207 Uehara, Nishihara, Okinawa 903-0215, Japan

Full list of author information is available at the end of the article
}

virus has never caused a documented pandemic. Consequently, most epidemiological studies have focused on influenza A infection rather than influenza B infection.

In temperate regions, it is well established that epidemics of influenza A often occur during the winter [3]. However, in tropical and subtropical regions, influenza A infections have been observed throughout the year [9]. In contrast, seasonal information for influenza B is limited and epidemic patterns of influenza B can vary among investigations [10, 11]. Again, most climate analysis studies have focused on influenza A infection; data regarding influenza B infection is lacking considerably $[10,11]$. 
In Japan, the universal healthcare system supports all citizens to visit outpatient clinics, or the emergency departments of general hospitals, without referral. As a result, patients with mild disease, including upper respiratory tract infection, often visit general hospitals directly. In many cases, attending physicians suspecting an influenza-like illness (ILI) will use a rapid antigen test (RAT), which can detect influenza A and B separately [12]. In Okinawa, four representative general hospitals aggregate and report the weekly results of the RATs. Using this routine surveillance data, our investigative team previously reported the epidemic pattern of influenza A viral infection and its relationship to climatic conditions $[13,14]$. This retrospective study aims to characterize the epidemic patterns of influenza B by combining the aforementioned RAT reported results with the nation-wide influenza surveillance data accumulated between 2007 and 2014. Our multicenter approach includes patients from Okinawa, an important surveillance region in subtropical Japan. Influenza A in this study is included for comparison.

\section{Methods}

Dataset 1- local surveillance of influenza virus infection by rapid antigen test (Additional file 1)

The clinical laboratories of four representative general hospitals, Naha City Hospital (470 beds), Okinawa Red Cross Hospital (314 beds), Okinawa Prefectural Nanbu Medical Center (434 beds), and Urasoe General Hospital (311 beds), in the capital city of Okinawa, reported the results of the RATs for influenza virus weekly to the Clinical Laboratory Center of the Medical Association. Data accessed from the Clinical Laboratory Center of the Medical Association included only the total number of tests administered and the number of positive influenza A, B, or A/B co-infected results. Patient information and symptomatology were not available.

\section{Dataset 2- Japanese national sentinel surveillance data (Additional file 1)}

The Japanese national influenza surveillance is conducted in approximately 5000 sentinel healthcare facilities throughout Japan; 55 facilities are within the Okinawa Prefecture [15]. Data from these 55 sentinel healthcare facilities within Okinawa was extracted from the Infectious Diseases Weekly Reports, published by the National Institute of Infectious Diseases in Japan [16, 17]. This surveillance dataset includes the age distribution of patients diagnosed with an influenza infection based on either a positive RAT result and/or the presence of symptoms from ILI. Influenza-like illness is defined with four criteria as follows; 1) acute onset of symptoms, 2) high fever, 3) upper respiratory symptoms, and 4) general symptoms such as malaise, headache, and myalgia. Patients with either a positive RAT result or who meet all four ILI criteria can be added to the database.
Evaluation of age distribution of influenza A or B infection Weekly disease patterns were monitored in dataset 1 retrospectively. A week in which influenza A or B cases accounted for more than $90 \%$ of all positive influenza cases was subsequently defined as an "epidemic week" for either influenza A or B, respectively. Weeks with no predominant influenza type (i.e., A to B ratio was 1:1) were not considered as "epidemic weeks" and were excluded from age distribution analysis. Weeks determined as "epidemic weeks" in dataset 1 were matched to the corresponding week in dataset 2. Patient age distribution from those corresponding weeks was extracted from dataset 2 for the sentinel sites located within the Okinawa Prefecture. A visual representation for this method is provided in Additional file 2: Table S1.

\section{Geographic and climatic background}

Okinawa Island is located in the East China Sea approximately $640 \mathrm{~km}$ south of the rest of Japan, and roughly 500 $\mathrm{km}$ north of Taiwan. Possessing a diverse native population, a large population of semi-permanent foreigners, and an ample tourist trade, Okinawa has proven its worth as a surveillance site for the monitoring of circulating respiratory viruses prior to epidemic outbreaks in mainland Japan [18]. Average temperatures are $18{ }^{\circ} \mathrm{C}$ in winter and $28{ }^{\circ} \mathrm{C}$ in summer. The island's subtropical climate supports a dense forest and a rainy season occurring in the late spring. Daily climate data including ambient temperature and relative humidity was retrieved from the Japanese Meteorological Agency website (http://www.jma.go.jp/jma/ index.html) [19]. Weekly climate variables were calculated using the retrieved data.

\section{Statistical analysis}

The duration of infectious activity was assessed using the maximum proportion of cases during consecutive weeks and the minimum number of weeks during which there were at least $80 \%$ positive RAT results, a method previously described by Caini S, et al. [20]. Positive RAT cases and climate variables were evaluated by Spearman's correlation coefficient test (one sided). The datasets used in this study can be found within the additional files. Statistical analyses were performed using the SPSS software (version 20.0, IBM Tokyo, Japan).

\section{Results}

From January 2007 to March 2014, 168,874 RATs were performed within the four representative hospitals. The use of RATs diagnosed a total of 37,309 and 7277 influenza $A$ and influenza $B$ infections, respectively. The incidence of influenza B infection was lowest for 2010, the year following the influenza pandemic of 2009. The annual number of influenza B positive cases ranged from 107 (0.9\% of all RATs in 2010) to 1940 (9.5 \% of all RATs in 2011), as shown in Table 1. Co-infection with both A and 
Table 1 Result of rapid antigen tests performed in four general hospitals in Okinawa

\begin{tabular}{lllll}
\hline Year & Number of tests performed & Influenza A positive & Influenza B positive & Influenza A/B positive \\
\hline 2007 & 19,229 & $5143(26.7 \%)$ & $623(4.4 \%)$ & $6(0.04 \%)$ \\
2008 & 13,089 & $1878(14.3 \%)$ & $797(7.1 \%)$ & $4(0.04 \%)$ \\
2009 & 41,962 & $12,741(30.4 \%)$ & $1153(3.9 \%)$ & $18(0.06 \%)$ \\
2010 & 13,825 & $2508(18.1 \%)$ & $107(0.9 \%)$ & $2(0.02 \%)$ \\
2011 & 24,468 & $4128(16.9 \%)$ & $1940(9.5 \%)$ & $7(0.04 \%)$ \\
2012 & 27,148 & $5284(19.5 \%)$ & $1353(6.2 \%)$ & $4(0.02 \%)$ \\
2013 & 18,494 & $2475(13.4 \%)$ & $728(4.5 \%)$ & $0(0.00 \%)$ \\
2014 (Jan. to Mar.) & 10,659 & $3152(29.6 \%)$ & $576(7.7 \%)$ & $1(0.01 \%)$ \\
Total & 168,874 & $37,309(22.1 \%)$ & $7277(4.3 \%)$ & $42(0.03 \%)$ \\
\hline
\end{tabular}

The annual number of rapid antigen tests (RAT) performed and reported positive results for influenza $A$, B, and A/B co-infected patients in four representative hospitals in Okinawa, Japan

$\mathrm{B}$ was not common. Figure 1 shows the percentage of positive cases for influenza B was less than influenza A. Epidemic analysis of influenza B revealed bimodal peaks observed in March and May (data not shown). The middle $80 \%$ of influenza B infections were seen from week 11 (middle March) to week 30 (end July), in contrast to the middle $80 \%$ of non-pandemic strain influenza A cases occurring from week 51 (end December) to week 13 (middle March) of the following year. Influenza A infection, in seasons other than winter, was primarily attributed to the pandemic strain of 2009 (Fig. 2).

Epidemic curves from the two datasets were aligned and analyzed to define at risk age groups (Fig. 3). Overall, patients aged 0 to 9 years old or 10 to 19 years old more frequently visit the sentinel sites included in dataset 2 (lower) during periods of influenza B outbreaks reported in dataset 1 (upper). An additional analysis of age categories was conducted to validate these results. Age categories of the patients affected by either influenza type were determined using the method depicted in Additional file 2: Table S1. This method subsequently defined, 173 weeks with influenza A cases $>90 \%(148,244$ assumed influenza A cases) and 78 weeks with influenza B cases $>90 \%$ (13,606 assumed influenza B cases). Age dynamics from weeks categorized as influenza B weeks show 5 to 9 yearolds (28\%) and 10 to 14 year-olds (25\%) as the predominant age groups seeking healthcare for confirmed or probable cases of influenza. The same age patterns were not observed for influenza A weeks (Fig. 4).

Finally, the impact of climate variables on the proportion of positive influenza A or B results was evaluated on a weekly basis. As shown in Figs. 5 and 6, a higher proportion of influenza A positive cases were observed during periods of lower ambient temperature and lower

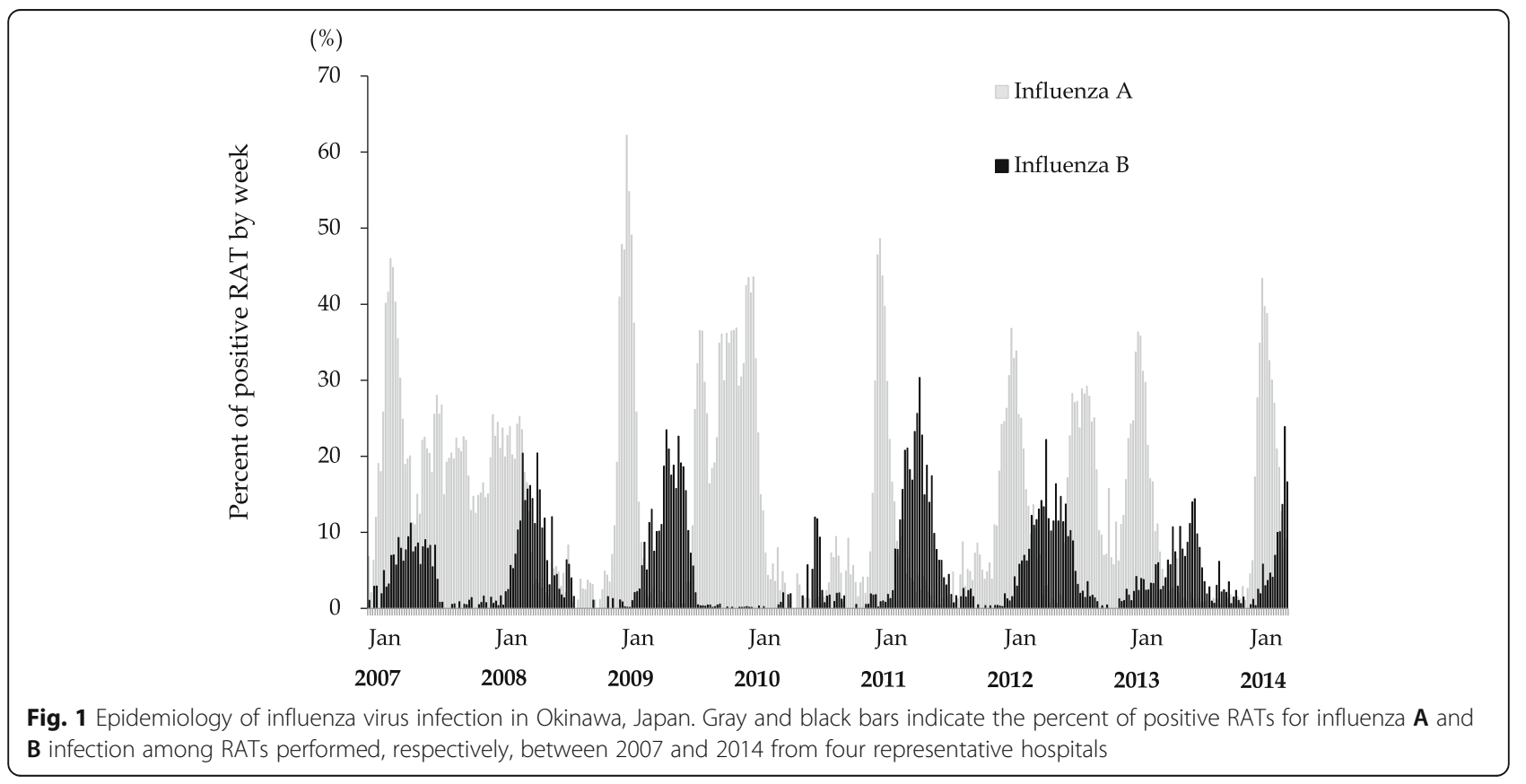




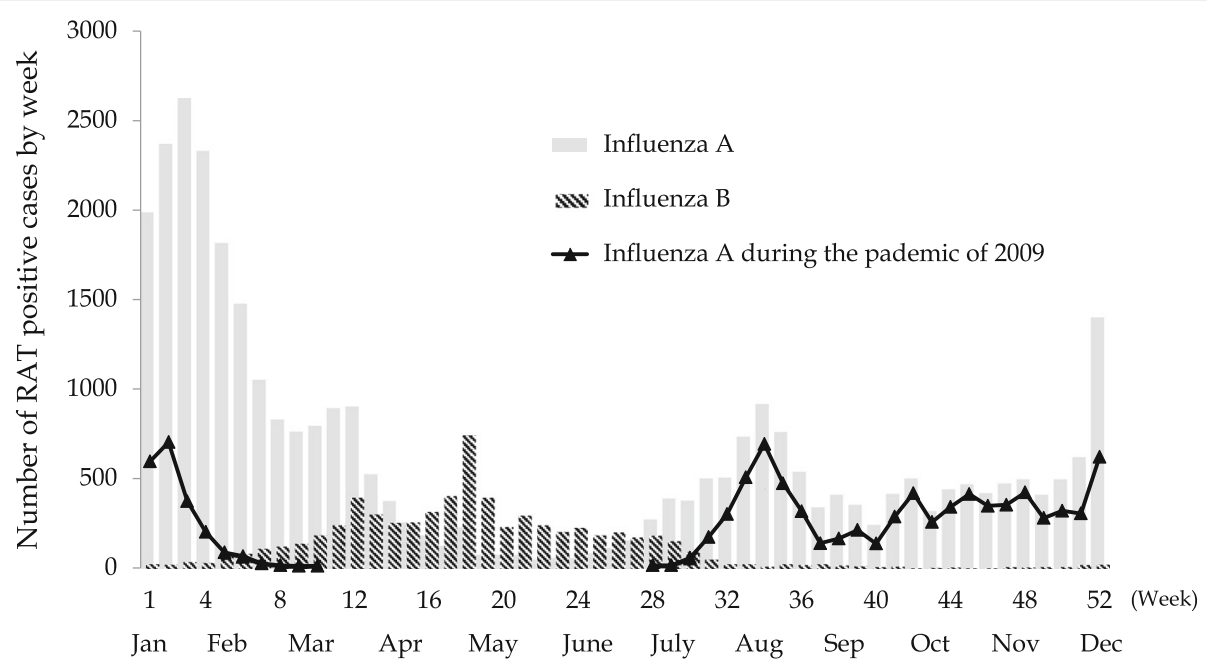

Fig. 2 Pooled number of positive cases of influenza infection by week. Gray and hashed bars indicate the number of positive cases determined by rapid antigen test (RAT) for influenza A and B infection, respectively. Cases are pooled for each week from 2007 until 2014 using the RAT results from four representative hospitals. Triangles indicate the number of influenza $\mathbf{A}$ infections determined by RAT during the pandemic year of 2009 from four representative hospitals (from June 2009 to March 2010)

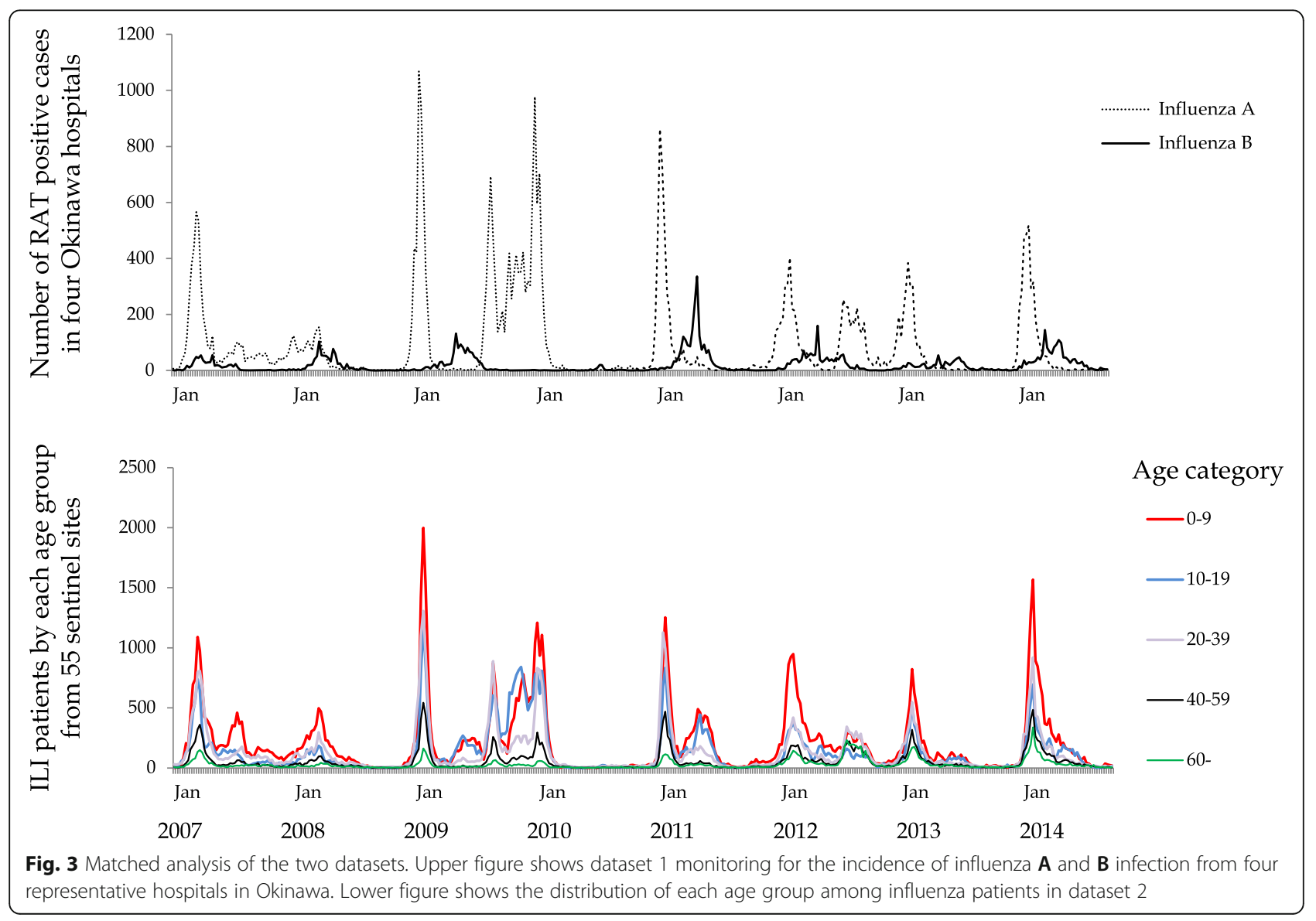




\section{Influenza A}

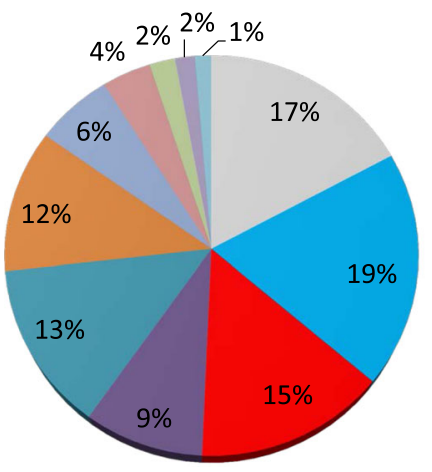

$0 \sim 4$
$40 \sim 49$
- $10 \sim 14$
$60 \sim 69$
Influenza B

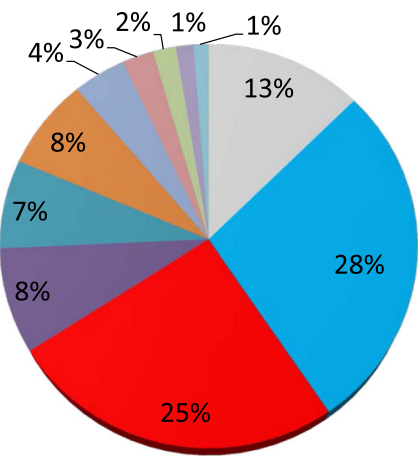

$\square 15 \sim 19$

$\square 70 \sim 79$

$\square 20 \sim 29$

- $30 \sim 39$

80

Age categories

Fig. 4 Age category distribution during epidemic weeks defined as influenza $\mathbf{A}$ or $\mathbf{B}$ infection. Epidemic weeks of influenza $\mathbf{A}$ or $\mathbf{B}$ were identified using the described method within dataset 1. Information regarding age distribution for all identified weeks was extracted from dataset 2. Assumed cases of influenza A and $\mathbf{B}$ totaled 148,244 and 13,606, respectively. Only patient data from the 55 sentinel sites located within Okinawa was used for age comparison

relative humidity. Alternatively, a high proportion of influenza B positive cases were observed during times of high relative humidity. No association between ambient temperature and influenza B could be determined.

\section{Discussion}

Okinawa's annual influenza B epidemics primarily occur between March and July and display bimodal peaks in March and May. Although Kikuchi et al. reported a 2006 epidemic of influenza B from May to June in Sapporo, Japan [6], epidemics of influenza $B$ infection are rarely reported from mainland Japan and other temperate regions. However, intermittent epidemics of influenza B have been reported from Taiwan [21]. Also, a recently published article by the Global Influenza B Study Team examined the cooperation and coordination of influenza $\mathrm{A}$ and $\mathrm{B}$ viruses. Together, influenza A and B's combined effect successfully extended the influenza season for countries located within

\section{Influenza A}

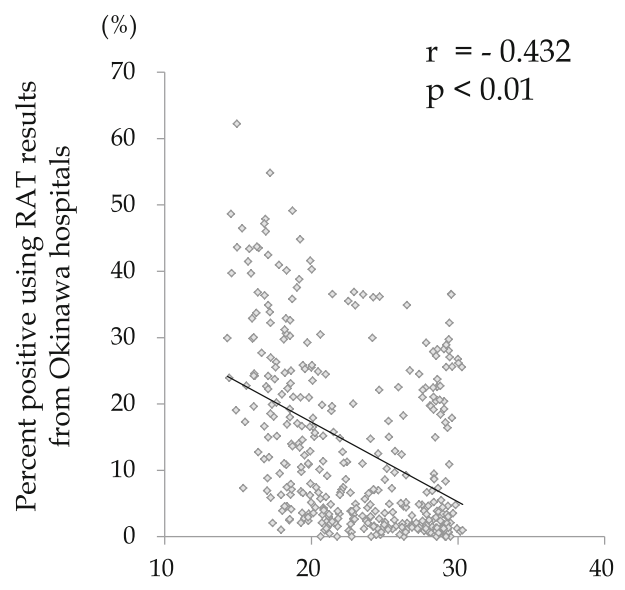

\section{Influenza B}

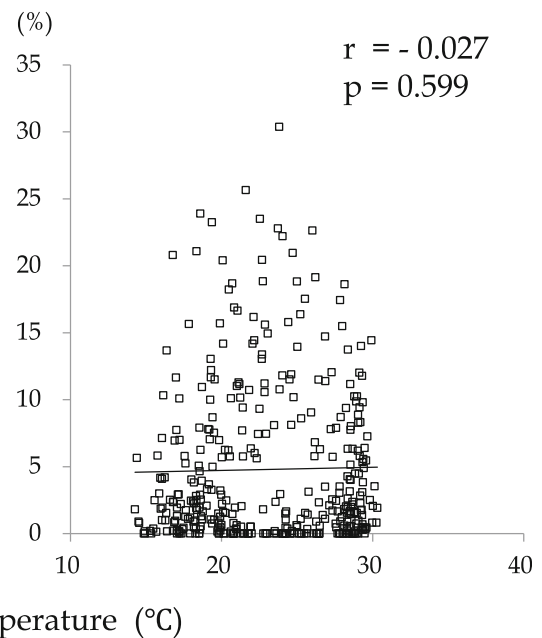

Fig. 5 Ambient temperature's effect on influenza infections in Okinawa, Japan. The relationship between ambient temperature and the percent of positive influenza $\mathbf{A}$ or $\mathbf{B}$ infection for RAT results performed in four representative hospitals was analyzed with Spearman rank correlation coefficient (one-sided). r; correlation coefficient, $p ; p$ value 
Influenza A

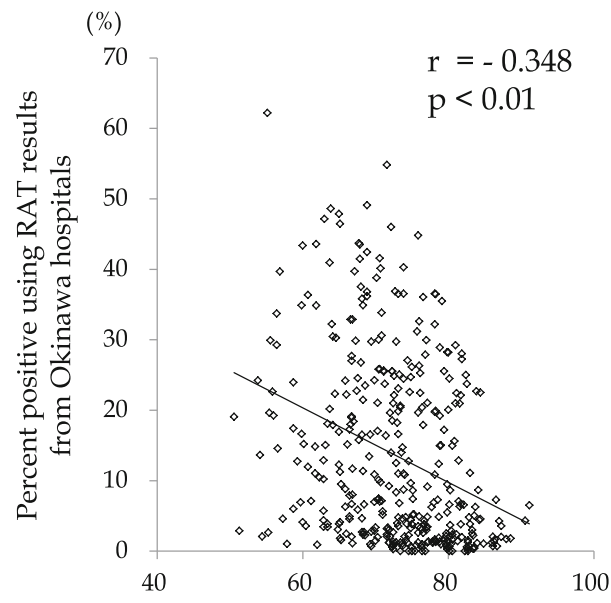

Influenza B

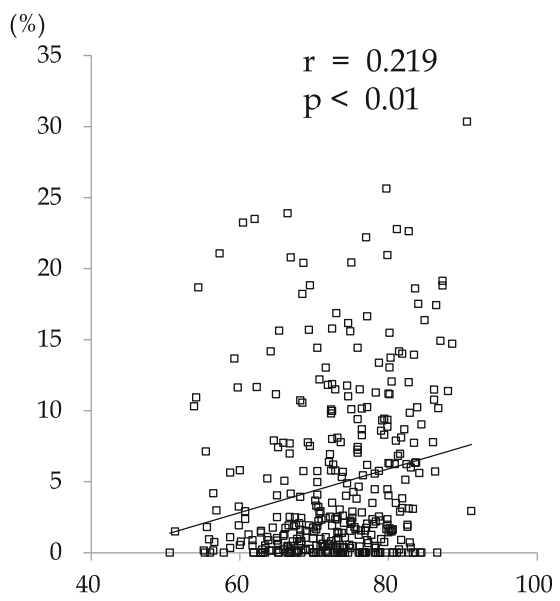

Relative humidity (\%)

Fig. 6 Relative humidity's effect on influenza infections in Okinawa, Japan. The relationship between relative humidity and the percent of positive influenza $\mathbf{A}$ or $\mathbf{B}$ infection for RAT results performed in four representative hospitals was analyzed with Spearman rank correlation coefficient

(one-sided). r; correlation coefficient, $p$; $p$ value

tropical and subtropical regions [20]. Similarly, the subtropical climate of Okinawa may enable the seasonal patterns of influenza infection observed in the present study.

It is common knowledge that cold, dry weather can produce favorable conditions for influenza A virus transmission [22-24]. It is also known that influenza A virulence is considered greater than that of influenza B virus due to genetic shift and drift $[2,25,26]$. Following a winter with influenza A infection in decline, it is possible, influenza B virus could more easily spread among those with weakened immunity. Additionally, the dynamics among viruses themselves can influence patterns of epidemics. As we have seen with the pandemic strain in 2009-2010, influenza A has the ability to suppress outbreaks of other respiratory viruses, particularly influenza $B$ [27]. Thus, the differences in pathogenicity between influenza A and B could also explain the seasonal patterns observed in Okinawa. Ultimately, the characteristics of influenza epidemics for tropical and subtropical countries are complex and still poorly understood. In reality, the interaction of several climatic and ecological drivers, including temperature, humidity, altitude precipitation, population density and cultural mores or gathering events could create the ideal setting for pervasive circulation.

In the present study, we were able to assess the association between the incidence of influenza A or B infection and limited climatic conditions. Influenza A virus remained significantly associated with cold and dry climate conditions [22-24]. However, the association between influenza B infection and climatic conditions was less distinctive. Our analysis confirmed a high percentage of influenza A positive cases were observed during periods of lower ambient temperature and lower relative humidity. On the other hand, a high percentage of influenza B cases were associated with high humidity. No link was observed between influenza B infection and temperature (Figs. 5 and 6). In this study, we use relative humidity not specific humidity, which may limit the accuracy of our results. Previous investigations from our team have also used relative humidity $[13,14]$, and the standardization of the method was important to compare this current study with previous data. Recent articles have reported specific humidity is a better predictor of epidemics of influenza [28, 29]. Therefore, further analysis using specific humidity is needed to clarify the relationship between influenza $B$ and humidity in Okinawa. Since multiple factors are involved in determining region-specific epidemic patterns of influenza infection [30], more prospective epidemiological studies, including analyses of meteorological factors, are needed to understand the circumstances found in Okinawa and other tropical and subtropical regions.

During influenza B epidemics, our results showed the primary patient group visiting healthcare facilities in Okinawa was school-age children (Figs. 3 and 4). Alternatively, influenza A affects a much broader age range, suggesting that this phenomenon is unique to influenza $B$ infection. The pathogenicity of influenza A virus is considerable and all age-groups are susceptible to influenza A infection $[3,16]$. As a result of this virulence, differences in prevalence among each age-group might become less distinct. Influenza B virus cannot cause massive epidemics among all age-groups due to its relatively weak virulence. 
Other reports also show that influenza B patients were younger than influenza A patients $[10,11]$.

Healthcare seeking behavior may also account for the different patterns observed among the age groups. It is well known that the school-age population can easily spread influenza infections [31-34]. Working adults might be less likely to visit a clinic with only mild symptoms due to influenza B infection. However, parents of school age children may be more likely to seek immediate care for a sick child, regardless of severity of symptoms. Additionally, acquired immunity within the host may account for the observed differences among the age groups. Adults with repeated exposure to influenza $B$ may be less susceptible to the disease, since mutation rates for influenza $B$ are not as rapid as influenza A [35].

This study has some limitations. First, sample collection and laboratory analysis were performed externally. The results of RATs performed in four representative general hospitals in Okinawa is routinely accumulated and summarized on a weekly basis. In addition, Japan has a nation-wide influenza surveillance system with weekly reports available to all physicians. All data was received either through the local prefectural influenza reporting system or through the Japanese national influenza reporting system. Due to this external processing, patient details such as gender, co-morbidities, and accompanying symptoms could neither be accessed nor analyzed. Nevertheless, the Western Pacific Region Global Surveillance and Response System has set precedent that surveillance data for ILI symptoms and datasets based on influenza viral detection can be synchronized [25]. Our data replicates this method and confirmed the epidemic curves of the two different datasets overlapped in Okinawa (Fig. 3).

A second limitation is the lack of standardization in RAT protocols and manufacturers. Due to retrospective nature of this study and the diversity of hospitals reporting data, there was no way to standardize which RAT test was implemented. Okinawa serves as an important surveillance point to monitor the circulating strains of influenza $[13,18]$. Therefore, physicians in Okinawa are perpetually aware and use RATs for patients with ILI, regardless of the season. As a result, usage of RATs for patients with ILI is higher than average. Overall, the diagnostic accuracy of RATs sold in Japan is high. At one point in 2013, 17 different RATs for influenza virus were available in Okinawa. According to the included manufacturer's documents, the sensitivity and specificity of RATs for influenza A virus, when compared with a gold standard viral culture, ranged from 87.9 to $100 \%$ and 88.8 to $100 \%$, respectively. Detection of influenza B virus ranged from 80.4 to $100 \%$ and 90.7 to $100 \%$, respectively. However, RAT results should be expected to show significant heterogeneity due to differences in patient age, virus type and brand variety, all of which can affect test results. Furthermore, some studies describe lower sensitivity for detection of influenza $B$ infection than that of influenza A infection [12, 36-39]. This decrease in sensitivity may cause even lower rates of detection for influenza $B$, due to increased false negative results in some populations.

Lastly, this study did not investigate any patient factors with influence on immunity, mainly, vaccination. In Japan, a quadrivalent vaccine against influenza viruses was introduced in the 2015-16 season. However, the administration of influenza vaccine is generally recommended before winter, and it is doubtful if the efficacy of vaccine against influenza B infection lasts until spring. In the future, discussions regarding the efficacy and the cost effectiveness of the single quadrivalent vaccine compared to a bi-annual vaccine schedule should be considered if a decrease in influenza infections is not achieved in Okinawa.

\section{Conclusion}

In Okinawa, influenza B epidemics primarily occur from March until July with bimodal peaks in March and May and predominately affect school-age children. Our data suggests high relative humidity may increase rates of influenza B infection. No novel information was discovered regarding our comparator, influenza A, from this study. Additional studies within the surveillance site of Okinawa, or other subtropical regions are needed to confirm our results and expand our knowledge regarding the epidemiology of influenza B.

\section{Additional files}

Additional file 1: Dataset 1. Positivity rate. Dataset 2. Patiant's age (XLSX $161 \mathrm{~kb}$ )

Additional file 2: Table 1. Example weeks from dataset 1 and dataset 2 post-alignment. Sample weeks shown here are to serve as a visual representation of the method outlined to evaluate age distribution. Dataset 1 (left) and dataset 2 (right) were combined and aligned following the selection of "epidemic weeks". A week in which influenza A or B cases accounted for more than $90 \%$ of all positive influenza cases was defined as an epidemic week. In the year 2007, you see an example of a defined influenza A epidemic week, whereas the year 2011 is a representative influenza B epidemic week. The week from 2013 displays a typical week which was removed from our age distribution analysis because neither influenza A nor B was dominant (>90 \%). (PPTX 66 kb)

\section{Abbreviations}

ILI: Influenza-like illness; RAT: Rapid antigen test

\section{Acknowledgements}

Not applicable.

Funding

None.

Authors' contributions

YI planed this study, collected and analyzed data, and prepared manuscript. TK supervised this project and prepared manuscript with YI and GP. FH organized this study, analyzed data. HM collected surveillance data. JF organized and supervised this project. All authors read and approved the final manuscript. 


\section{Availability of data and materials}

The datasets used in this study can be found within the additional files.

\section{Competing interests}

Authors declare that they have no conflict of interests.

\section{Consent for publication}

Not applicable.

\section{Ethics approval and consent to participate}

This study was approved by the Institutional Review of Board of the University of the Ryukyus. This was a retrospective study and data were anonymously collected. There was no additional disadvantage for the subjects.

\section{Author details}

'Department of Infectious Diseases, Respiratory, and Digestive Medicine, Graduate School of Medicine, University of the Ryukyus, 207 Uehara, Nishihara, Okinawa 903-0215, Japan. ${ }^{2}$ Department of Nursing, University of the Ryukyus Hospital, 207 Uehara, Nishihara, Okinawa 903-0215, Japan. ${ }^{3}$ Department of Respiratory Medicine, National Hospital Organization Okinawa Hospital, 3-20-14 Ganeko, Ginowan, Okinawa 901-2214, Japan. ${ }^{4}$ Clinical Laboratory Center, Medical Association of Naha City, 26-1 Higashimachi, Naha, Okinawa 900-0034, Japan.

Received: 5 February 2016 Accepted: 27 October 2016

Published online: 08 November 2016

\section{References}

1. Global Influenza Surveillance and Response System, http://www.who.int/ influenza/gisrs_laboratory/en/. Accessed 20 Dec 2015.

2. Fineberg HV. Pandemic preparedness and response-Lessons from the H1N1 influenza of 2009. N Engl J Med. 2013;370:1335-42.

3. Lofgren E, Fefferman NH, Naumov YN, Gorski J, Naumova EN. Influenza seasonality: underlying causes and modeling theories. J Virol. 2007;81:5429-36.

4. Bedford T, Riley S, Barr IG, Broor S, Chadha M, Cox NJ, et al. Global circulation patterns of seasonal influenza viruses vary with antigenic drift. Nature. 2015;523:217-20.

5. Finkelman BS, Viboud C, Koelle K, Ferrari MJ, Bharti N, Grenfell B. Global Patterns in Seasonal Activity of Influenza A/H3N2, A/H1N1, and B from 1997 to 2005, Viral Coexistence and Latitudinal Gradients. PLoS One. 2007;2: e1296.

6. Kikuchi M, Yamamoto M, Yoshida Y, Miyashita T, Fujita K. Epidemics of Influenza from Winter to Summer in the 2005/06 Season in Sapporo, Japan. Jpn J Infect Dis. 2007;60:152-3.

7. Cottrell SL, Moore C, Dexter L, Thomas DR, Salmon RL. Unusually high impact of influenza B during the early 2012-2013 influenza season in Wales_epidemiology and clinical analysis of the first 100 cases. Influenza Other Respir Viruses. 2013;7:1013-6.

8. Chiu SC, Lin JH, Wang HC, Wu HS, Chang HW, Lin YC, et al. Molecular epidemiologic and clinical characteristics of influenza B- associated complications among hospitalized patients during an outbreak in Taiwan. Int J Infect Dis. 2014;23:94-100.

9. Park AW, Glass K. Dynamic patterns of avian and human influenza in East and Southeast Asia. Lancet Infect Dis. 2007:7:543-8.

10. Glezen WP, Schmier JK, Kuehn CM, Ryan KJ, Oxford J. The burden of influenza B: a structured literature review. Am J Public Health. 2013;103:e43-51.

11. Caini S, Huang QS, Ciblak MA, Kusznierz G, Owen R, Wangchuk S, et al. Epidemiological and virological characteristics of influenza B: results of the Global Influenza B Study. Influenza Other Respir Viruses. 2015;9:3-12.

12. Tanei M, Yokokawa $H$, Murai $K$, Sakamoto R, Amari Y, Boku S, et al. Factors influencing the diagnostic accuracy of the rapid influenza antigen detection test (RIADT): a cross-sectional study. BMJ Open. 2014;4:e003885.

13. Higa F, Naka M, Tatayama M, Haranaga S, Yara S, Koide M, et al. Epidemiology of influenza from 2007 to 2008 in the Naha area, Okinawa prefecture: surveillance of rapid antigen test results. Jpn J Infect Dis. 2009; 62:399-401.

14. Iha Y, Higa F, Sunagawa S, Naka M, Cash HL, Miyagi K, et al. Effect of climatic conditions on epidemic patterns of influenza in Okinawa, Japan, during pandemic of 2009: Surveillance of rapid antigen test results. Jpn Infect Dis. 2012;65:295-300.
15. Okabe N, Yamashita K, Taniguchi K, Inouye S. Influenza surveillance system of Japan and acute encephalitis and encephalopathy in the influenza season. Pediatrics Int. 2000;42:187-91.

16. Infectious Disease Surveillance center, http://www.nih.go.jp/niid/ja/flu-map. html. Accessed 10 Jun 2016.

17. Okinawa prefecture Infectious Diseases Surveillance Center, http://www idsc-okinawa.jp/butainflu.html. Accessed 15 Jan 2016.

18. Suzuki Y, Taira K, Saito R, Nidaira M, Okano S, Zaraket H, et al. Epidemiologic study of influenza infection in Okinawa, Japan, from 2001-2007: changing patterns of seasonality and prevalence of amantadine- resistant influenza $A$ virus. J Clin Microbiol. 2009:47:623-9.

19. Japanese Meteorological Agency, http://www.jma.go.jp/jma/index.html. Accessed 30 Apr 2014

20. Caini S, Andrade W, Badur S, Balmaseda A, Barakat A, Bella A, et al. Temporal Patterns of Influenza A and B in Tropical and Temperate Countries: What Are the Lessons for Influenza Vaccination? PLos One. 2016;11:e0155089.

21. Yang JR, Huang YP, Chang FY, Hsu LC, Lin YC, Huang HY, et al. Phylogenetic and evolutionary history of influenza B viruses, which caused a large epidemic in 2011-2012, Taiwan. PLoS One. 2012;7:e47179.

22. Shaman J, Kohn M. Absolute humidity modulates influenza survival, transmission, and seasonality. Proc Natl Acad Sci U S A. 2009;106:3243-8.

23. Lowen AC, Mubareka S, Steel J, Palese P. Influenza virus transmission is dependent on relative humidity and temperature. PLoS Pathog. 2007;3:1470-6.

24. Minhaz Ud-Dean SM. Structural explanation for the effect of humidity on persistence of airborne virus: seasonality of influenza. J Theor Biol. 2010;264:822-9.

25. Western Pacific Region Global Influenza Surveillance and Response System. Epidemiological and virological characteristics of Influenza in the western Pacific region of the World health organization, 2006-2010. PLoS One. 2012;7:e37568.

26. Fouchier RA, Munster V, Wallensten A, Bestebroer TM, Herfst S, Smith D, et al. Characterization of a Novel Influenza A Virus Hemagglutinin Subtype (H16) Obtained from Black-Headed Gulls. J Virol. 2005;79:2814-22.

27. van Asten L, Bijkerk P, Fanoy E, van Ginkel A, Suijkerbuijk A, van der Hoek W, et al. Early occurrence of influenza A epidemics coincided with changes in occurrence of other respiratory virus infections. Influenza Other Respir Viruses. 2016:10:14-26.

28. Shaman J, Pitzer VE, Viboud C, Grenfell BT, Lipsitch M. Absolute humidity and the seasonal onset of influenza in the continental United States. PLoS Biol. 2010;23:e1000316.

29. Shoji M, Katayama K, Sano K. Absolute humidity as a deterministic factor affecting seasonal influenza epidemic in Japan. Tohoku J Exp Med. 2011; 224:251-6.

30. Tamerius J, Nelson MI, Zhou SZ, Viboud C, Miller MA, Alonso WJ. Global influenza seasonality: reconciling patterns across temperate and tropical regions. Environ Health Perspect. 2011;119:439-45.

31. Kawai S, Nanri S, Ban E, Inokuchi M, Tanaka T, Tokumura M, et al. Influenza vaccination of schoolchildren and influenza outbreaks in a school. Clin Infect Dis. 2011;53:130-6.

32. Talbot HK, Poehling KA, Williams JV, Zhu Y, Chen Q, Mcnabb P, et al. Influenza in Older Adults: Impact of Vaccination of School Children. Vaccine. 2009:27:1923-7.

33. Cauchemez S, Van Kerkhove MD, Archer BN, Cetron M, Cowling BJ, Grove P, et al. School closures during the 2009 influenza pandemic: national and local experiences. BMC Infect Dis. 2014;14:207.

34. Chi CY, Wang SM, Lin CC, Wang HC, Wang JR, Su IJ, et al. Clinical features of children infected with different strains of influenza B in southern Taiwan. Pediatr Infect Dis J. 2008;27:640-5.

35. Nobusawa E, Sato K. Comparison of the mutation rates of human influenza A and B viruses. J Virol. 2006;80:3675-8.

36. Chartrand C, Leeflang MM, Minion J, Brewer T, Pai M. Accuracy of rapid influenza diagnostic tests: a meta-analysis. Ann Intern Med. 2012;156:500-11.

37. Jacobus $\mathrm{CH}$, Raja AS. How accurate are rapid influenza diagnostic tests? Ann Emerg Med. 2013;61:89-90.

38. Hurt AC, Alexander R, Hibbert J, Deed N, Barr IG. Performance of six influenza rapid tests in detecting human influenza in clinical specimens. J Clin Virol. 2007;39:132-5

39. Cho CH, Woo MK, Kim JY, Cheong S, Lee CK, An SA, et al. Evaluation of five rapid diagnostic kits for influenza A/B virus. J Virol Methods. 2013;187:51-6. 